\title{
Preferential orientation of biological apatite crystallite in original, regenerated and diseased cortical bones
}

\author{
Takayoshi NAKANO, ${ }^{\dagger}$ Takuya ISHIMOTO, Jee-Wook LEE and Yukichi UMAKOSHI \\ Course of Materials Science and Engineering, Division of Materials and Manufacturing Science, Graduate School of Engineering, \\ Osaka University, 2-1, Yamada-Oka, Suita, Osaka 565-0871
}

\begin{abstract}
The bone mechanical function depends on both bone quantity and quality corresponding dominantly to bone mineral density (BMD: density of biological apatite) and other factors, respectively. BMD is correlated with bone strength but accounts for only $60-70 \%$ of the variance in the ultimate strength of bone tissue. Thus, new parameters representing bone quality have been investigated so far. Bone has a well-organized microstructure on the nano-scale level and is composed of mineral biological apatite (BAp) and collagen (Col) fibril, providing reinforcement and pliability, respectively. Because BAp crystallizes in an anisotropic hexagonal lattice, mechanical properties of a BAp crystallite should depend on the crystal orientation. Thus, preferential orientation of the BAp $c$-axis along the extended collagen fibrils in hard tissues must be closely related to the mechanical function of bone and is also utilized as a possible index for evaluating bone quality. In this study, BAp orientation was analyzed as a parameter of bone quality in hard tissues under various conditions such as original, regenerated and diseased bones. Finally, we can conclude that the degree of BAp orientation is a useful parameter to evaluate in vivo stress distribution, nano-scale microstructure and the related mechanical function, the regenerative process of the regenerated bone and to diagnose bone diseases such as osteoarthritis, etc.
\end{abstract}

Key-words: Biological apatite (BAp), Texture, Microbeam X-ray diffraction, Tissue engineering, Regeneration, Osteoarthritis (OA), Bone quality

[Received October 5, 2007; Accepted January 11, 2008] @2008 The Ceramic Society of J apan

1. Introduction

The bone mechanical function depends on both bone quantity and quality corresponding dominantly to bone mineral density and the integrity of the internal architecture, respectively. BMD is correlated with bone strength and is conventionally used for bone diagnosis but accounts for only $60-70 \%$ of the variance in ultimate strength of bone tissue. ${ }^{1)}$ Thus, new parameters representing bone quality have been investigated so far.

Bone has a well-organized microstructure on the nanoscale level and is composed of a mineral phase of biological apatite (BAp) and an organic phase of collagen ( $\mathrm{Col}$ ) fibril, providing reinforcement and pliability, respectively, as shown in Fig. 1. ${ }^{2)}$,3) Because BAp crystallizes in an anisotropic hexagonal lattice, mechanical properties of a BAp crystallite should depend on the crystal orientation. ${ }^{2)-5}$ ) Thus, preferential orientation of the BAp $c$-axis along the extended collagen fibrils in hard tissues must be closely related to the mechanical function of bone and is also utilized as a possible index for evaluating bone quality.

In this study, we clarified correlations among in vivo stress distribution, anisotropy of the BAp/Col orientation and the mechanical function, evaluated by bone shape, BAp texture and Young's modulus, respectively, in the original intact, regenerated and diseased hard tissues.

\section{Experimental procedure}

Bone samples under various conditions such as mature intact cortical, regenerated and diseased bones, were prepared. X-ray diffraction analysis for the crystallographic

\footnotetext{
† Corresponding author: nakano@mat.eng.osaka-u.ac.jp
}

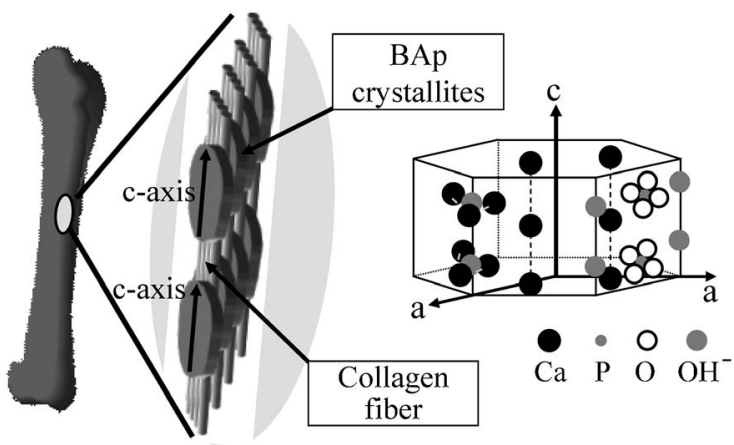

Fig. 1. Bone microstructure and crystal structure of biological apatite (BAp). The $c$-axis of BAp is roughly aligned in the same direction as collagen. ${ }^{3)}$ At the same time, the hexagonal crystalbased structure has an extremely high anisotropic ion arrangement.

approach to the constituent BAp crystallites was performed using the microbeam X-ray diffractometer system. ${ }^{6}$ ) The incident beam was focused onto a circular beam spot of 100 $\mu \mathrm{m}$ in diameter. BMD was measured by a peripheral quantitative computed tomography ( $\mathrm{pQCT})$. Bone tissue was detected as a CT value of more than $267 \mathrm{mg} / \mathrm{cm}^{3}$.

Data are presented as the mean value with standard deviation (SD). SD is expressed by an error bar. A Student's t-test was used for comparison between the groups. Correlations among the analyzed parameters were assessed by Pearson's correlation coefficient. A $P$-value of less than 0.05 was judged to be statistically significant. 


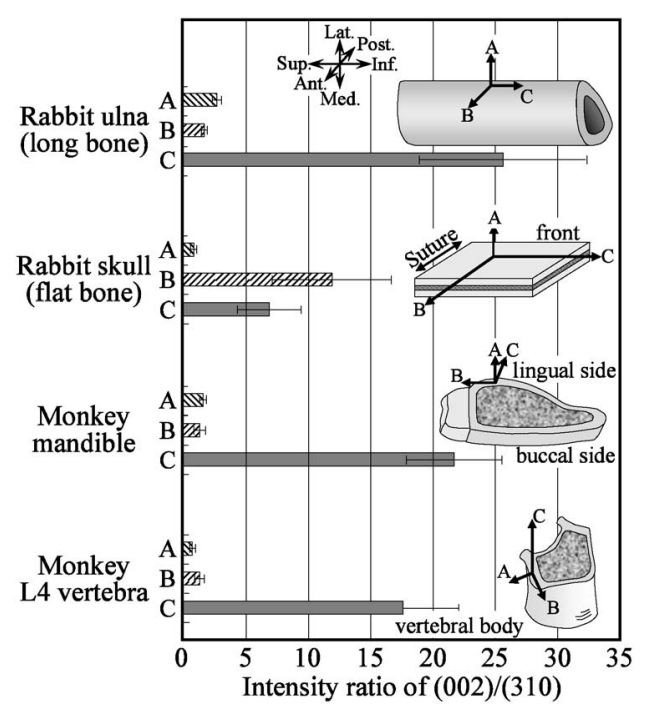

Fig. 2. Preferential orientation of the BAp $c$-axis in typical mature intact cortical bones. Higher value in the intensity ratio means higher degree of BAp preferential orientation of $c$-axis. The random orientation of BAp corresponds to a value of two. Measurements were done along $\mathrm{A}, \mathrm{B}$, and $\mathrm{C}$ direction for each bone, and unique BAp texture was found depending on the anatomic site and bone shape.

\section{Results and discussion}

Original intact bones from several anatomic sites such as a rabbit ulna, a rabbit skull, a monkey mandible and a monkey vertebra exhibit unique texture of the BAp crystallites relating to the arrangement of Col fibrils. Preferential orientation of anisotropic BAp crystallites in typical cortical bones changes depending on the anatomic site and bone shape. For example, the rabbit ulna as a long bone and the monkey vertebra have a one-dimensional texture of the BAp $c$-axis along the $\mathrm{C}$ direction as described in Fig. 2 meanwhile, the skull bone as a flat bone shows two-dimensional BAp texture along the $\mathrm{B}$ and $\mathrm{C}$ directions. The dentulous mandible basically exhibits a one-dimensional texture of the BAp $c$-axis along the $\mathrm{C}$ direction parallel to the mesiodistal axis in the anatomic site where no biting stress is directly distributed. The degree of the BAp $c$-axis orientation was calculated by the (002) and (310) diffractions in the microbeam X-ray profiles because these diffractions are relatively isolated from the other diffractions which are found at Bragg angles of $25.9^{\circ}$ and $39.8^{\circ}$, respectively. Figure 3 shows an example of the microbeam X-ray diffraction profile from the monkey vertebral body along the $\mathrm{C}$ direction parallel to the craniocaudal axis. The preferential orientation of the BAp $c$-axis corresponds to the in vivo stress distribution and BAp $c$-axis tends to orientate along the loading stress direction in the original bones as shown in Fig. 2. ${ }^{6}$

In order to clarify how bone tissue is regenerated and the function is then improved in the defective portion, we analyzed the recovery process of not only BMD but also preferential BAp orientation and the related Young's modulus (data is not shown here) by using a rabbit ulnar osteotomy model. Figure 4 shows the relationship between $\mathrm{BMD}$ and the $c$-axis of BAp in the longitudinal direction. Normal bones have BMD of about $1200 \mathrm{mg} / \mathrm{cm}^{3}$ and BAp orientation, as relative intensity between (002) and (310)

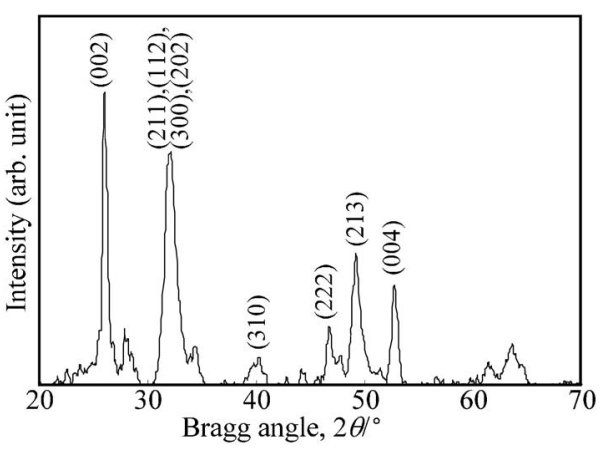

Fig. 3. Typical microbeam X-ray diffraction profile of the monkey 4 th vertebral body taken along the $\mathrm{C}$ axis described in Fig. 2 .

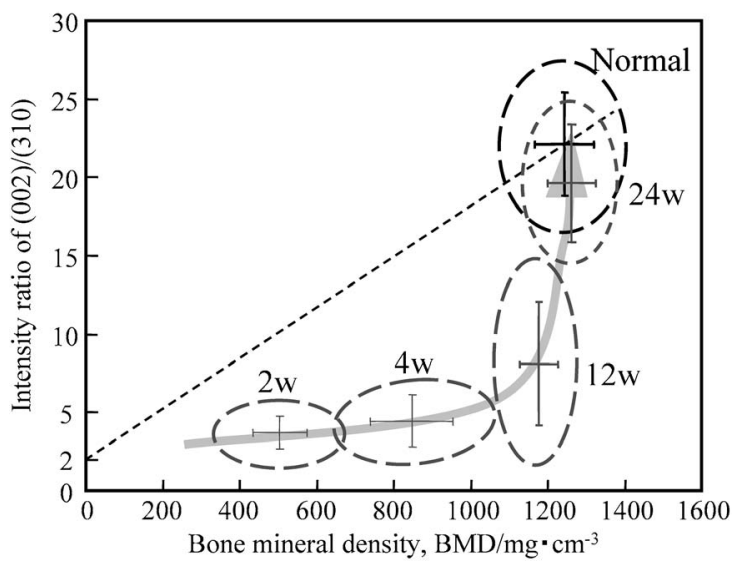

Fig. 4. Recovery process of BMD and BAp preferential orientation (the intensity ratio of $(002) /(310)$ ) along the longitudinal direction in the regenerated ulna. The BAp preferential orientation recovered behind the BMD restoration.

diffraction peaks, of 20 to $25 .^{7)-9)}$ When bone density and BAp orientation recover at the same rate, this is represented by the dotted line in the middle of the figure. However, in actuality bone density recovers at a faster rate than BAp orientation; the rate of BAp orientation recovery is initially slow, but then accelerates. This suggests that the formula "bone density=BAp orientation" cannot be established, which means the recovery of bone microstructures such as the BAp orientation cannot be determined only from BMD. Thus, reloading on the regenerated portion caused by BMD restoration was suggested to accelerate to produce the appropriate BAp preferential orientation. We also clarified the correlations among in vivo stress distribution, the BAp orientation and mechanical function, especially ultimate stress $^{10)}$ and Young's modulus, in the regenerated hard tissues.

Moreover, changes in bone quantity and bone quality were investigated in diseased bone tissue such as osteoarthritis (OA), osteoporosis and osteopetrosis. Focusing on OA compared with a normal knee joint, for example, BMD of subchondral bone is significantly higher and subchondral bone thickness is clearly thicker in OA than those in the normal tissue (Fig. 5(a)). ${ }^{11}$ Furthermore, the preferential orientation of the BAp $c$-axis along the normal direction of subchondral bone in OA is significantly higher (Fig. 5(b)) 
(a)

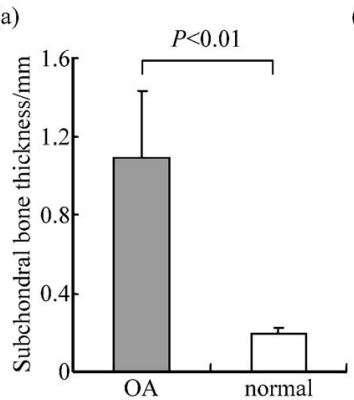

(b)

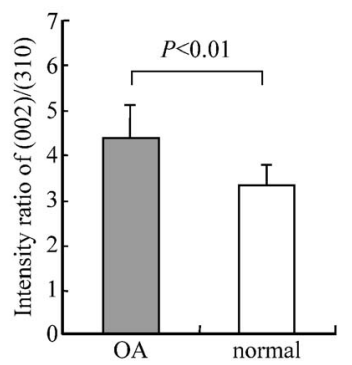

Fig. 5. Comparison of subchondral bone thickness and BAp orientation (intensity ratio) at the close contact portion of knee joint between $\mathrm{OA}$ and normal. The measurements were repeated 9 times in every knee, and ten OA and five normal knee joints were analyzed for the statistical analysis.

than that in normal subchondral bone tissue because of the stress concentration in the diseased portion without a cartilage. ${ }^{12)}$ It seems that the hardening in OA subchondral bone is reflected by not only the proliferative increment of bone tissue but also an excess degree of BAp preferential orientation compared with the normal tissue.

The BAp orientation was finally concluded to be one of the most important indices to evaluate in vivo stress distribution, nano-scale microstructure and the related mechanical function, the regenerative process of the regenerated bone and to diagnose bone diseases such as osteoarthritis, osteoporosis etc.

\section{Summary}

The orientation degree of BAp crystallite in the representative direction is expected to serve as a bone quality parameter with wide application in the medical field in the near future such as analysis of the progression and severity of bone diseases, evaluation of regeneration in regenerative bone tissues, judgment of the effectiveness of drugs for osteoporosis and other bone diseases, and design of biomaterials based on bone microstructure because the BAp

orientation is closely related to the bone functions.

Acknowledgements This work was supported by funds from the "Priority Assistance of the Formation of Worldwide Renowned Centers of Research-The 21st Century COE Program and The Global COE program (Project: Center of Excellence for Advanced Structural and Functional Materials Design)" and a Grant-in-Aid for Scientific Research and Development from the Ministry of Education, Culture, Sports, Science and Technology of Japan. The work was partly supported by the Industrial Technology Research Program (Project: 03A47002) in 2003 from the New Energy and Industrial Technology Development Organization (NEDO) of Japan and by CREST, Japan Science and Technology Agency.

\section{References}

1) B. D. Synder, S. Piazza, W. T. Edwards and W. C. Hayes, Calcif. Tissue Int., 53, S14-S22 (1993).

2) J. C. Elliot, "Structure and chemistry of the apatites and other calcium phosphates", Elsevier, Amsterdam (1994).

3) W. J. Landis, Bone, 16, 533-544 (1995).

4) N. Sasaki, N. Matsushima, T. Ikawa, H. Yamaura and A. Fukuda, J. Biomech., 22, 157-164 (1989).

5) T. Nakano, Y. Tabata and Y. Umakoshi, "Texture and Bone reinforcement", Encyclopedia of Materials: Science and Technology-Updates, Ed. by K. H. J. Buschow, R. W. Cahn, M. C. Flemings, E. J. Kramer, P. Veyssiere et al. (2005) pp. MS2061-1.

6) T. Nakano, K. Kaibara, Y. Tabata, N. Nagata, S. Enomoto, E. Marukawa and Y. Umakoshi, Bone, 31, 479-487 (2002).

7) T. Nakano, K. Kaibara, Y. Tabata, N. Nagata, S. Enomoto, E. Marukawa and Y. Umakoshi, "Tissue Engineering for Therapeutic Use 6”, Ed. by Y. Ikada, Y. Umakoshi and T. Hotta, Elsevier Science, Amsterdam (2002) pp. 95.

8) T. Ishimoto, T. Nakano, Y. Umakoshi, M. Yamamoto and Y. Tabata, Mater. Sci. Forum, 512, 261-264 (2006).

9) T. Nakano, T. Ishimoto, J.-W. Lee, Y. Umakoshi and Y. Tabata, Mater. Sci. Forum, 539-543, 675-680 (2007).

10) T. Ishimoto, T. Nakano, Y. Umakoshi, M. Yamamoto and Y. Tabata, Mater. Sci. Forum, 561-565, 1451-1454 (2007).

11) J. W. Lee, T. Nakano, A. Kobayashi, K. Takaoka, Y. Tabata and Y. Umakoshi, Phosph. Res. Bull., 17, 83-84 (2004).

12) W. J. Anderst and S. Tashman, J. Biomech., 36, 1291-1299 (2003). 